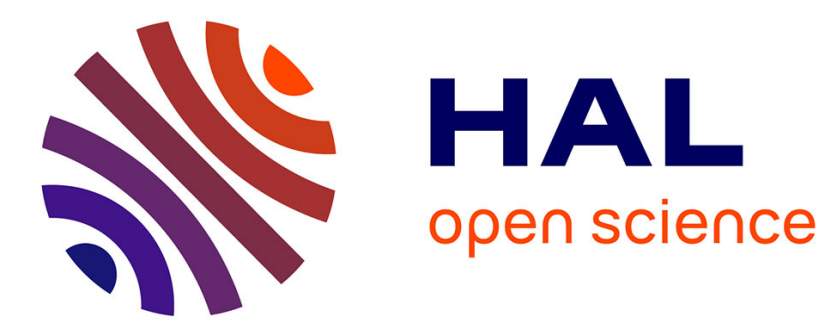

\title{
Control of Dual-Airfoil Airborne Wind Energy systems based on nonlinear MPC and MHE
}

\author{
Mario Zanon, Greg Horn, Sebastien Gros, Moritz Diehl
}

\section{To cite this version:}

Mario Zanon, Greg Horn, Sebastien Gros, Moritz Diehl. Control of Dual-Airfoil Airborne Wind Energy systems based on nonlinear MPC and MHE. European Control Conference (ECC), 2014, Strasbourg, France. pp.1801 - 1806, 10.1109/ECC.2014.6862238 . hal-01068909

\section{HAL Id: hal-01068909 https://hal.inria.fr/hal-01068909}

Submitted on 26 Sep 2014

HAL is a multi-disciplinary open access archive for the deposit and dissemination of scientific research documents, whether they are published or not. The documents may come from teaching and research institutions in France or abroad, or from public or private research centers.
L'archive ouverte pluridisciplinaire HAL, est destinée au dépôt et à la diffusion de documents scientifiques de niveau recherche, publiés ou non, émanant des établissements d'enseignement et de recherche français ou étrangers, des laboratoires publics ou privés. 


\title{
Control of Dual-Airfoil Airborne Wind Energy Systems Based on Nonlinear MPC and MHE
}

\author{
Mario Zanon, Greg Horn, Sébastien Gros and Moritz Diehl
}

\begin{abstract}
Airborne Wind Energy (AWE) systems generate energy by flying a tethered airfoil across the wind flow at a high velocity. Tethered flight is a fast, strongly nonlinear, unstable and constrained process, motivating control approaches based on fast Nonlinear Model Predictive Control (NMPC) and state estimation approaches based on Moving Horizon Estimation (MHE). Dual-Airfoil AWE systems, i.e. systems with two airfoils attached to a Y-shaped tether have been shown to be more effective than systems based on a single airfoil. This paper proposes a control scheme for a dual-airfoil AWE system based on NMPC and MHE and studies its performance in a realistic scenario based on state-of-the-art turbulence models.

Keywords : airborne wind energy, fast NMPC and MHE, trajectory tracking, dual airfoils
\end{abstract}

\section{INTRODUCTION}

Over the last years, conventional wind turbines have grown in size and mass up to a scale at which the major challenges are posed by the structural loads [1], [2]. The main idea behind Airborne Wind Energy (AWE) is to eliminate all the elements of the system which are not essential for power extraction, resulting in a much lighter structure that only involves an airfoil tethered to the ground. In this configuration, higher altitudes can be reached and the swept area is not fixed by the structure of the system, but can be optimized so as to maximise the extracted power. The system is thus free to operate in previously inaccessible portions of the wind field, where higher wind resources can be found.

The potential of this technology has been established in theory [3]. Several architectures have been proposed for AWE systems, ranging from a single tethered airfoil to complex multi-airfoil structures. In [4], the power extracted by a single tethered airfoil was compared to the one extracted by two airfoils attached to a Y-shaped tether. The study showed the potential of the dual airfoil configuration, which was able to extract more power than the single airfoil for all considered airfoil dimensions.

Tethered airfoils are strongly nonlinear, constrained unstable systems subject to strong perturbations (e.g. wind

M. Zanon, G. Horn, S. Gros and M. Diehl are with the Optimization in Engineering Center (OPTEC), K.U. Leuven, Kasteelpark Arenberg 10, B-3001 Leuven-Heverlee, Belgium. mario.zanondesat.kuleuven. be

* This research was supported by Research Council KUL: PFV/10/002 Optimization in Engineering Center OPTEC, GOA/10/09 MaNet and GOA/10/11 Global real- time optimal control of autonomous robots and mechatronic systems. Flemish Government: IOF / KP / SCORES4CHEM, FWO: $\mathrm{PhD} /$ postdoc grants and projects: G.0320.08 (convex MPC), G.0377.09 (Mechatronics MPC); IWT: PhD Grants, projects: SBO LeCoPro; Belgian Federal Science Policy Office: IUAP P7 (DYSCO, Dynamical systems, control and optimization, 2012-2017); EU: FP7- EMBOCON (ICT248940), FP7-SADCO ( MC ITN-264735), ERC ST HIGHWIND (259 166), Eurostars SMART, ACCM. gusts). Nonlinear model predictive control (NMPC) is an advanced optimization-based control technique able to deal with nonlinear systems while satisfying the given constraints (e.g. avoid stall). Moving horizon estimation (MHE) is an optimization-based observer able to take into account the full nonlinear dynamics of the system. For single airfoils, control schemes based on NMPC have been proposed in [5], [6] and based on both NMPC and MHE have been proposed in [7], [8], [9].

To the authors' knowledge, no control scheme has been proposed so far for dual airfoils attached to a Y-shaped tether. In this paper, a MHE and NMPC based control scheme for dual airfoils is presented. Each airfoil is modeled as a rigidbody, 6-DOF object interacting with the air mass. The poweroptimal periodic reference trajectory is computed using the method proposed in [10].

When using general purpose solvers, the computational burden of solving the MHE and NMPC dynamic optimization problem can be excessive for real-time applications, especially when dealing with fast mechanical systems such as tetherd airfoils. In order to address this issue, the realtime iteration (RTI) scheme has been proposed in [11]. This scheme was successfully implemented in an extension to the open-source software ACADO that exports tailored, efficient C code [12]

This paper is organized as follows. The process model is presented in Section II and the control scheme based on MHE and NMPC is proposed in Section III. Simulation results are presented in Section IV. Future developments and conclusions are proposed in Section V.

\section{SySTEM MOdeL}

\section{A. Airfoil Kinematics}

In a similar way as in [4], the system is modeled as a tree structure with three nodes coinciding with the joint position (node $i=0$ ) and the airfoil center of mass (nodes $i=1,2$ ). Each airfoil is considered as a rigid body having 6 degrees of freedom (DOF). An orthonormal right-hand reference frame $E$ is chosen s.t. a) the wind is blowing in the $E^{1}$-direction, b) the vector $E^{3}$ is opposed to the gravitational acceleration vector $g$.

A right-hand orthonormal reference frame $e_{i}$ is attached to each airfoil node $(i=1,2)$ s.t. a) the basis vector $e_{i}^{x}$ spans the wing longitudinal axis, pointing in the forward direction and is aligned with the wing chord, b) the basis vector $e_{i}^{z}$ spans the vertical axis, pointing in the upward direction. Each 
airfoil attitude is given by the rotation matrix

$$
R_{i}=\left[\begin{array}{lll}
e_{i}^{x} & e_{i}^{y} & e_{i}^{z}
\end{array}\right],
$$

where vectors $e_{i}^{x, y, z}$ are given in $E$.

The position of node $i$ in the reference frame $E$ is given by $r_{i}=\left[x_{i}, y_{i}, z_{i}\right]^{T}$. Each tether segment $i$ is approximated as a rigid link of length $l_{i}$ that constrains $r_{i}$ to be on the manifold defined by

$$
\begin{aligned}
c_{0} & =\frac{1}{2}\left(\left\|r_{0}\right\|_{2}^{2}-l_{0}^{2}\right)=0, \\
c_{i} & =\frac{1}{2}\left(\left\|r_{i}+R_{i} r_{\mathrm{T}}-r_{0}\right\|_{2}^{2}-l_{i}^{2}\right)=0, \quad i=1,2,
\end{aligned}
$$

where $r_{\mathrm{T}}$ is the position of the tether attachment point in $e_{i}$.

Because the center of mass of the airfoils does not coincide with the tether attachment point, translational and rotational dynamics are not separable. Using Lagrange mechanics and the methods described in [13], [14] and introducing the algebraic states $\lambda=\left[\lambda_{0}, \lambda_{1}, \lambda_{2}\right]^{T}$, the equations of motion can be described as index- 1 differential algebraic equations (DAE):

$$
\begin{aligned}
& {\left[\begin{array}{cc}
M & G \\
G^{T} & 0
\end{array}\right]\left[\begin{array}{c}
\ddot{r} \\
\dot{\omega} \\
\lambda
\end{array}\right]=F, \quad \ddot{r}=\left[\begin{array}{c}
\ddot{r}_{0} \\
\ddot{r}_{1} \\
\ddot{r}_{2}
\end{array}\right], \quad \dot{\omega}=\left[\begin{array}{c}
\dot{\omega}_{1} \\
\dot{\omega}_{2}
\end{array}\right]} \\
& a_{i}=\left[a_{i}^{\mathrm{a}} a_{i}^{\mathrm{e}} a_{i}^{\mathrm{r}} \kappa_{i}\right]^{T}, \quad \dot{a}_{i}=u_{i}, \quad i=1,2 \\
& R_{i}(0)^{T} R_{i}(0)-I_{3}=0, \quad \dot{R}_{i}=R_{i}\left(\omega_{i}\right)_{\times}, \quad i=1,2 \\
& c_{i}(0)=0, \quad \dot{c}_{i}(0)=0, \quad i=1,2,3
\end{aligned}
$$

where $I_{3}$ is the $3 \times 3$ identity matrix, $\left(\omega_{i}\right)_{\times} \in S O(3)$ is the skew symmetric matrix of the rotational velocity $\omega_{i}$ and

$$
\begin{aligned}
& M=\left[\begin{array}{ccccc}
\sum_{i=0}^{3} \xi_{i} & \frac{1}{2} \xi_{1} & \frac{1}{2} \xi_{2} & 0 & 0 \\
\frac{1}{2} \xi_{1} & \xi_{1}+m_{1} I_{3} & 0 & 0 & 0 \\
\frac{1}{2} \xi_{2} & 0 & \xi_{2}+m_{2} I_{3} & 0 & 0 \\
0 & 0 & 0 & J_{1} & 0 \\
0 & 0 & 0 & 0 & J_{2}
\end{array}\right] \\
& G=\left[\begin{array}{ccc}
r_{0} & \nabla_{r_{0}} c_{1} & \nabla_{r_{0}} c_{2} \\
0 & \nabla_{r_{1}} c_{1} & 0 \\
0 & 0 & \nabla_{r_{2}} c_{2} \\
0 & 2 P_{R_{1}}\left(\nabla_{R_{1}} c_{1}\right) & 0 \\
0 & 0 & 2 P_{R_{2}}\left(\nabla_{R_{2}} c_{2}\right)
\end{array}\right] \\
& F=\left[\begin{array}{c}
F_{0}-\frac{1}{2} g \mu_{0} l_{0} I_{3}-\sum_{i=1}^{2} \frac{1}{2} g \mu_{i} l_{i} I_{3} \\
F_{1}-\frac{1}{2} g \mu_{1} l_{1} I_{3}-g m_{1} I_{3} \\
F_{2}-\frac{1}{2} g \mu_{2} l_{2} I_{3}-g m_{2} I_{3} \\
T_{1}-\omega_{1} \times J_{1} \omega_{1} \\
T_{2}-\omega_{2} \times J_{2} \omega_{2} \\
\nabla_{r_{0}} \dot{c}_{0}^{T} \dot{r}_{0} \\
-\nabla_{r_{0}} \dot{c}_{1}^{T} \dot{r}_{0}-\nabla_{r_{1}} \dot{c}_{1}^{T} \dot{r}_{1}-2 P_{R_{1}}\left(\nabla_{R_{1}} \dot{c}_{1}\right)^{T} \omega_{1} \\
-\nabla_{r_{0}} \dot{c}_{2}^{T} \dot{r}_{0}-\nabla_{r_{2}} \dot{c}_{2}^{T} \dot{r}_{2}-2 P_{R_{2}}\left(\nabla_{R_{2}} \dot{c}_{2}\right)^{T} \omega_{2}
\end{array}\right],
\end{aligned}
$$

where $\xi_{i}=\frac{1}{3} \mu_{i} l_{i} I_{3}, \mu_{i}$ denotes the density of tether segment $i, m_{i}$ and $J_{i}$ are respectively the mass and rotational inertia of airfoil $i$. The sum of the forces applied to node $i$ is denoted by $F_{i}$. Similarly, the sum of the torques applied to airfoil $i$ is denoted by $T_{i}$. The projcetion opertation $P_{R}(\cdot)$ is defined as $P_{R}(A)=U\left(R^{T} A\right)$, and $U$ is the unskew operator

$U\left(\left[\begin{array}{lll}a_{11} & a_{12} & a_{13} \\ a_{21} & a_{22} & a_{23} \\ a_{31} & a_{32} & a_{33}\end{array}\right]\right)=\frac{1}{2}\left[\begin{array}{l}a_{32}-a_{23} \\ a_{13}-a_{31} \\ a_{21}-a_{12}\end{array}\right], \quad U\left(a_{\times}\right)=a$.

Note that, with this formulation, the tether tension is readily given by $\Gamma_{i}=\lambda_{i} l_{i}$.

\section{B. Airfoil Aerodynamic Forces}

Introducing the relative velocity $v_{i}$, i.e. the velocity of the airfoil w.r.t. the air mass given by:

$$
v_{i}=\dot{r}_{i}-w_{i},
$$

where $w_{i}=\left[w_{i}^{x}, w_{i}^{y}, w_{i}^{z}\right]^{T} \in \mathcal{W} \subset \mathbb{R}^{3}$ is the wind velocity field at the location of airfoil $i$. The relative velocity in the airfoil reference frame is given by $V_{i}=R_{i}^{T} v_{i}$. The angle of attack $\alpha_{i}$ and sideslip angle $\beta_{i}$

$$
\alpha_{i}=-\tan \left(\frac{V_{i}^{z}}{V_{i}^{x}}\right), \quad \beta_{i}=\tan \left(\frac{V_{i}^{y}}{V_{i}^{x}}\right) .
$$

The aerodynamic forces and torques are given by

$$
\begin{aligned}
F_{i}^{\mathrm{A}} & =\frac{1}{2} \rho A\left\|v_{i}\right\|\left(C_{i}^{\mathrm{L}} v_{i} \times e_{i}^{y}-C_{i}^{\mathrm{D}} v_{i}\right), \\
T_{i}^{\mathrm{A}} & =\frac{1}{2} \rho A\left\|v_{i}\right\|^{2}\left[\begin{array}{lll}
C_{i}^{\mathrm{R}} & C_{i}^{\mathrm{P}} & C_{i}^{\mathrm{Y}}
\end{array}\right]^{T},
\end{aligned}
$$

where the aerodynamic coefficients of lift $C_{i}^{\mathrm{L}}, \operatorname{drag} C_{i}^{\mathrm{D}}$, roll $C_{i}^{\mathrm{R}}$, pitch $C_{i}^{\mathrm{P}}$ and yaw $C_{i}^{\mathrm{Y}}$ are functions of $\alpha_{i}, \beta_{i}, \omega_{i}$ and of the control surfaces $a_{i}^{\mathrm{a}}, a_{i}^{\mathrm{e}}, a_{i}^{\mathrm{r}}[15]$.

The drag due to the onboard turbines is given by

$$
F_{i}^{\mathrm{G}}=-\kappa_{i}\left\|v_{i}\right\| v_{i}
$$

where $\dot{\kappa}_{i}=u_{i}^{\kappa}$ and $u_{i}^{\kappa}$ is a control variable. This model assumes that the generated force is opposed to the local velocity.

\section{Tether Drag}

To compute the tether drag, let's consider tether $i$ connecting nodes $i$ and $k$. The tether drag model assumes that an infinitesimal portion $l_{i} \mathrm{~d} \sigma$ of tether $i$ located at position $r_{k i}(\sigma)=\sigma r_{i}+(1-\sigma) r_{k}, \sigma \in[0,1]$ generates an infinitesimal drag force $\mathrm{d} F_{k i}$ given by:

$$
\begin{aligned}
\mathrm{d} F_{k i}(\sigma) & =F_{k i}(\sigma) \mathrm{d} \sigma=-\frac{1}{2} \rho d_{k} l_{k} C_{\mathrm{T}}^{\mathrm{D}}\left\|v_{T}\right\| v_{T} \mathrm{~d} \sigma \\
v_{T} & =\sigma \dot{r}_{i}+(1-\sigma) \dot{r}_{k}-\left[\begin{array}{lll}
W(z) & 0 & 0
\end{array}\right]^{T} \\
z & =\sigma z_{i}+(1-\sigma) z_{k},
\end{aligned}
$$

where $C_{\mathrm{T}}^{\mathrm{D}}$ is the drag coefficient of a cylinder. An infinitesimal displacement of point $r_{k i}(\sigma)$ is given by $\delta r_{k i}(\sigma)=$ $\sigma \delta r_{i}+(1-\sigma) \delta r_{k}$, therefore the contribution of the drag of tether $k$ to the generalized force acting on node $i$ is given by:

$$
F_{k, i}^{\mathrm{T}}=\int_{0}^{1} \sigma F_{k i}(\sigma) \mathrm{d} \sigma
$$


and conversely, the contribution of the drag of tether $k$ to the generalized force acting on node $k$ is given by:

$$
F_{k, k}^{\mathrm{T}}=\int_{0}^{1}(1-\sigma) F_{k i}(\sigma) \mathrm{d} \sigma .
$$

It should be observed that this formulation accounts for the total forces and moments acting on the system due to tether drags. Note that integrals (2) and (3) have to be evaluated numerically. In this paper, a trapezoidal quadrature is used with $M=6$ points. The contribution of the tether drags to the generalized forces acting on the generalized coordinates $r_{i}$ is given by:

$$
F_{i}^{\mathrm{T}}=F_{i, i}^{\mathrm{T}}+\sum_{k \in N(i)} F_{k, i}^{\mathrm{T}},
$$

where $N(i)$ associates to each node $i$ the set of nodes connected to it through a tether.

\section{Wind Turbulence Model}

Similarly to [8], [16], it is assumed that the wind field at the position of each airfoil $i$ is given by

$$
w_{i}=w_{i}^{\mathrm{t}}+w_{i}^{\ell}, \quad i=1,2,
$$

i.e. it is the superposition of a turbulent windfield $w_{\mathrm{t}}$ and a laminar logarithmic windshear model blowing in the $x$ direction

$$
w_{i}^{\ell}(z)=\left[\begin{array}{lll}
w_{0} \frac{\log \left(z_{i} / z_{\mathrm{r}}\right)}{\log \left(z_{0} / z_{\mathrm{r}}\right)} & 0 & 0
\end{array}\right], \quad i=1,2,
$$

where $w_{0}$ denotes respectively the wind velocity at height $z_{0}$ and $z_{\mathrm{r}}$ denotes the terrain roughness.

For control and estimation purposes, it is proposed here to use a rather simple turbulence model, given by

$$
\dot{w}_{i}^{\mathrm{t}}=-\frac{w_{i}^{\mathrm{t}}}{\tau}+u_{i}^{\mathrm{t}}, \quad i=1,2,
$$

where $u_{i}^{\mathrm{t}}$ is the forcing term in this first-order differential equation, here modeled as a pseudo-control. While in the estimation problem the optimizer is free to choose the term $u_{i}^{\mathrm{t}}$ so as to best fit the measurements, in the control problem, these modes are uncontrollable and, for the prediction horizon, the forcing term is assumed to be 0 .

While elaborate stochastic wind models exist in the literature, no turbulence model has been developed specifically for AWE systems.

In the following, the process dynamics and the process initial conditions will be put in the form:

$$
\mathcal{M}(X)\left[\begin{array}{c}
\dot{X} \\
\lambda
\end{array}\right]=f(X, \mathcal{U}), \quad C(X(0))=0,
$$

where $f$ and $\mathcal{M}$ and $C$ lump together the process dynamics and consistency conditions given by (1). The control vector is given by $\mathcal{U}=\left[u_{1}, u_{2}, u_{1}^{\mathrm{t}}, u_{2}^{\mathrm{t}}\right] \in \mathbb{R}^{14}$ and the state

\begin{tabular}{|c|c|c|c|c|c|}
\hline Variable & Description & Unit & Bounds & Variable & Weight \\
\hline$a_{i}^{\mathrm{a}}$ & Aileron & deg & {$[-15,15]$} & $r_{i}$ & $8 \mathrm{e}-8$ \\
\hline$a_{i}^{\mathrm{e}}$ & Elevator & deg & {$[-18.3,18.3]$} & $\dot{r}_{i}$ & $1.5 \mathrm{e}-6$ \\
\hline$a_{i}^{\mathrm{r}}$ & Rudder & deg & {$[-11.5,11.5]$} & $R_{i}$ & $6 e-8$ \\
\hline$u_{i}^{\mathrm{a}}$ & Aileron rate & $\mathrm{deg} / \mathrm{s}$ & {$[-13.2,13.2]$} & $\omega_{i}$ & $6 e-8$ \\
\hline$u_{i}^{\mathrm{e}}$ & Elevator rate & $\mathrm{deg} / \mathrm{s}$ & {$[-1.7,1.7]$} & $a_{i}^{\mathrm{a}}, a_{i}^{\mathrm{e}}, a_{i}^{\mathrm{r}}$ & 0.1 \\
\hline$u_{i}^{\mathrm{r}}$ & Rudder rate & $\mathrm{deg} / \mathrm{s}$ & {$[-11.5,11.5]$} & $\kappa_{i}$ & $9.4 \mathrm{e}-6$ \\
\hline$C_{i}^{\mathrm{L}}$ & Lift coefficient & - & {$[-1,1]$} & $u_{i}^{\mathrm{a}}, u_{i}^{\mathrm{e}}, u_{i}^{\mathrm{r}}$ & $9.4 e-6$ \\
\hline$\Gamma_{i}$ & Tether tension & $N$ & {$[0, \infty)$} & $u_{i}^{\kappa}$ & $9.4 \mathrm{e}-8$ \\
\hline
\end{tabular}
vector is given by $X=\left[r_{0}, \dot{r}_{0}, X_{1}, X_{2}\right] \in \mathbb{R}^{56}$ and $X_{i}=$ $\left[r_{i}, \dot{r}_{i}, R_{i}, \omega_{i}, a_{i}, w_{i}\right] \in \mathbb{R}^{25}$.

\begin{tabular}{lll}
\hline Sensor & Measurements & Standard deviation \\
\hline IMU & Linear acceleration & $0.25 \mathrm{~m} / \mathrm{s}^{2}$ \\
IMU & Angular velocity & $0.025 \mathrm{rad} / \mathrm{s}$ \\
Encoder & Tether length & $2.5 \cdot 10^{-3} \mathrm{~m}$ \\
Encoder & Control surface angle & $2.5 \cdot 10^{-4} \mathrm{rad}$ \\
\hline
\end{tabular}

TABLE I

AVAILABLE MEASUREMENTS

TABLE II

SYSTEM CONSTRAINTS AND WEIGHTS.

\section{E. Constraints}

The control surfaces deflections $a_{i}^{\mathrm{a}}, a_{i}^{\mathrm{e}}, a_{i}^{\mathrm{r}}$ and their $u_{i}^{\mathrm{a}}, u_{i}^{\mathrm{e}}, u_{i}^{\mathrm{r}}$ rates are constrained by the mechanical design of the airfoil and the chosen actuators.

In addition to the previous constraints, in order to keep the system in the region where the model assumptions are valid, further path constraints need to be added on the lift coefficients $C_{i}^{\mathrm{L}}$ to avoid stall and on the tether tension $\Gamma_{i}$, to make sure that the tethers are never in compression.

All constraints are summarized in Table II. In the following, all path constraints are lumped together as the inequality constraint function $h(\mathcal{X}, \mathcal{U}) \leq 0$.

\section{F. Available Sensors}

The airfoils are assumed to be both equipped with GPS, an inertial measurement unit (IMU), a pitot tube, a variometer, an air probe and control surface encoders. The IMU onboard each airfoil $i$ measures the linear accelerations $\ddot{q}_{i}=R_{i}^{T} \ddot{r}_{i}+$ $\left[\begin{array}{lll}0 & 0 & g\end{array}\right]^{T}$ and the rotational velocities $\omega_{i}$, given in frame $e_{i}$.

All available sensors are listed in Table III with the associated standard deviation $\sigma$. Because of actuator noise and inaccuracy, the control inputs computed by the controller, may not be perfectly implemented by the system and are also subject to noise. They are thus added as pseudomeasurements to the cost function. In the following, all measurements will be lumped together in the measurement function $y(X, \mathcal{U})$.

\section{CONTROLlER SYNTHESIS}

\section{A. Periodic Power Optimal Reference Trajectory}

In order to extract the maximum amount of energy from the airmass, the reference trajectory is computed by solving a 


\begin{tabular}{llcl}
\hline Sensor & Measurement & Variable & $\sigma$ \\
\hline IMU & Accelerations in frame $e_{i}$ & $\ddot{q}_{i}$ & $5 \mathrm{~cm} \cdot \mathrm{s}^{-2}$ \\
IMU & Angular velocities & $\omega_{i}$ & $1 \mathrm{deg} \cdot \mathrm{s}^{-1}$ \\
GPS & Absolute positions & $r_{i}$ & $0.1 \mathrm{~m}$ \\
GPS & Absolute velocities & $\dot{r}_{i}$ & $0.6 \mathrm{~m} \cdot \mathrm{s}^{-1}$ \\
Variometer & Absolute vertical velocity & $\dot{z}_{i}$ & $0.5 \mathrm{~m} \cdot \mathrm{s}^{-1}$ \\
Tether gauge & Tether tension & $\Gamma_{i}$ & $500 \mathrm{~N}$ \\
Pitot tube & Long. relative velocity & $V_{i}^{x}$ & $1 \mathrm{~m} \cdot \mathrm{s}^{-1}$ \\
Air Probe & AoA & $\alpha_{i}$ & $2.5 \mathrm{deg}$ \\
Air Probe & Side-slip angle & $\beta_{i}$ & $5 \mathrm{deg}$ \\
Encoders & Control surfaces deflections & $a_{i}^{\mathrm{a}}, a_{i}^{\mathrm{e}}, a_{i}^{\mathrm{r}}$ & $0.1 \mathrm{deg}$ \\
\hline
\end{tabular}

TABLE III

AVAILABLE SENSORS FOR MHE, WITH THE CORRESPONDING NOISE STANDARD DEVIATION $\sigma$

periodic optimal control problem (OCP) for a given reference windspeed $w_{0}$. The extracted power is given by

$$
P=\sum_{i=1}^{2} F_{i}^{\mathrm{G}} v_{i}
$$

In order to let the optimizer find the best trajectory, also the period $T$ of the orbit, the tether lengths $l_{i}$ and the tether diameters $d_{i}$ are treated as optimization variables and lumped

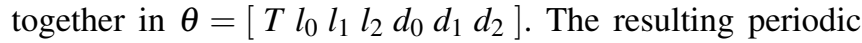
$\mathrm{OCP}$ is given by

$$
\begin{array}{cl}
\underset{\mathcal{U}(\cdot), X(\cdot), \lambda(\cdot), \theta}{\operatorname{minimize}} & \frac{1}{T} \int_{0}^{T} P d t, \\
\text { subject to } & \mathcal{M}(X, \theta)\left[\begin{array}{c}
\dot{X} \\
\lambda
\end{array}\right]=f(X, \mathcal{U}, \theta), \\
& X(0)=X(T), \quad h(X, \mathcal{X}, \theta) \leq 0,
\end{array}
$$

where the dependence on $\theta$ has been made explicit. The system being highly nonlinear, solving the periodic OCP (4) is nontrivial and a good initial guess is required in order to be able to solve it. In this paper, this difficulty has been addressed by using the relaxation technique proposed in [10] and successfully used in [17]. The computation of a suitable initial guess involves, itself, the solution of a simpler periodic OCP and a subsequent homotopy procedure [10].

\section{B. NMPC Formulation}

The receding horizon NMPC scheme is formulated using a least squares (LSQ) function penalizing the deviation of the process control inputs and states from the reference trajectories. The NMPC is based on repeatedly $(k=0,1, \ldots)$ solving the dynamic optimization problem:

$$
\begin{aligned}
\min _{\mathcal{U}(\cdot), X(\cdot), \lambda(\cdot)} & \frac{1}{2} \int_{t_{0}}^{t_{0}+T_{\mathrm{P}}}\left(\left\|X-X_{\mathrm{r}}\right\|_{Q}+\left\|\mathcal{U}-\mathcal{U}_{\mathrm{r}}\right\|_{R}\right) d t \\
\text { s.t. } & \mathcal{M}(X)\left[\begin{array}{c}
\dot{X} \\
\lambda
\end{array}\right]=f(X, \mathcal{U}), \\
& X\left(t_{0}\right)=\hat{X}\left(t_{0}\right), \quad h(X, \mathcal{U}) \leq 0,
\end{aligned}
$$

where $t_{k}=k T_{\mathrm{S}}$ and $T_{\mathrm{S}}$ is the NMPC sampling time, $T_{\mathrm{H}}$ the NMPC prediction horizon, $X_{\mathrm{r}}$ and $\mathcal{U}_{\mathrm{r}}$ define the state and control reference computed in (4) and $Q$ and $R$ are constant positive-definite weighting matrices. Vector $\hat{X}\left(t_{k}\right)$ is the process state estimated at time instant $t_{k}$ by solving the MHE problem (6).

Note that the process state estimate must satisfy the consistency condition $C\left(\hat{X}\left(t_{k}\right)\right)=0$. However, the consistency conditions are enforced by the state estimator, and therefore need not appear in the NMPC formulation.

\section{MHE Formulation}

The MHE scheme is formulated using a least squares (LSQ) function penalizing the deviation of the process control inputs and outputs from the measurements. The MHE estimate of the state $\hat{x}\left(t_{k}\right)$ is computed by repeatedly ( $k=$ $0,1, \ldots)$ solving the following dynamic optimization problem:

$$
\begin{aligned}
\min _{\mathcal{U}(\cdot), X(\cdot), \lambda(\cdot)} \frac{1}{2} \int_{t_{0}-T_{\mathrm{E}}}^{t_{0}}\|y(X, \mathcal{U})-\bar{y}\|_{Q_{\mathrm{E}}}^{2} d t, \\
\text { s.t. } \quad \mathcal{M}(X)\left[\begin{array}{c}
\dot{X} \\
\lambda
\end{array}\right]=f(X, \mathcal{U}), \\
C\left(X\left(t_{0}\right)\right)=0, \quad h(X, \mathcal{U}) \leq 0,
\end{aligned}
$$

where $y(X, \mathcal{U})$ is the system measurement function defined in Section II-F, $\bar{y}$ the corresponding set of measurements and $Q_{E}$ the corresponding covariance matrix.

\section{The Direct Multiple Shooting Method}

The system dynamics being unstable, problems (5) and (6) are best solved using simultaneous approaches [18] such as multiple shooting or collocation. In this paper, a uniform time discretization based on $N$ elements and such that $t_{0}<t_{1}<\ldots<t_{N}$ has been used in the framework of the Direct Multiple Shooting method [19]. The discrete-time formulation is thus obtained by independently integrating the system over each time interval $\left[t_{k}, t_{k+1}\right]$ and the path constraints are evaluated on the selected time grid. The basis functions for the control vector parametrization (CVP) have been chosen piecewise constant. The discretization of both (5) and (6) yields a least-squares NLP, that can be efficiently solved with the generalized Gauss-Newton method. The dimension of the resulting QP is then reduced by condensing [20].

\section{E. The Real Time Iterations with Shift}

The Real Time Iteration scheme is based on solving only a single full Newton-type iteration per sampling instant. The needed computations for the RTI scheme reduce to the computation of the sensitivities of the problem and the solution of a single QP. In this context, a clever initialization of the algorithms is crucial to guarantee contractivity of the scheme [11]. Based on the solution at the previous time instant, the initial guess for (5) and (6) is obtained by shifting the state and control vectors $X$ and $\mathcal{U}$ in time.

The initial value embedding consists in keeping the initial state in the optimization variables. This makes it possible to simulate the system, compute the sensitivities and thus run most of the computations before the current estimated state $\hat{X}\left(t_{0}\right)$ becomes available. Once the estimated state is 


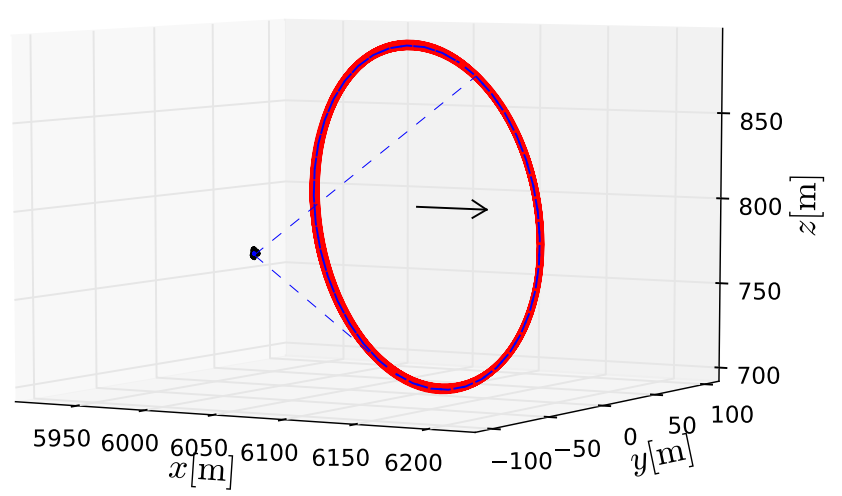

Fig. 1. Dual airfoil trajectory. The airfoil trajectories are displayed in red line and the joint position in thick black line. The reference trajectory is displayed in dashed blue line and the black arrow shows the reference wind direction.

available, the computation of the new controls can be done in a very short time by solving the QP prepared in the previous phase. See [11], [12], [21] for a detailed description of the RTI scheme.

\section{F. ACADO Code Generation}

In order to meet the real-time requirements, the code generation tool of ACADO [12], [22] has been used. This tool exports an efficient algorithm based on Direct Multiple Shooting and the RTI scheme. The resulting $\mathrm{C}$ code exploits the structure of the specific problem and avoids all irrelevant or redundant computations. The effectiveness of code generation in terms of reduced computational time, has been shown in [12], [23].

\section{Simulations}

\section{A. NMPC and MHE Tuning}

Both NMPC and MHE have been discretized with a sampling time $T_{\mathrm{S}}=125 \mathrm{~ms}$. The NMPC prediction horizon was set to $N_{\mathrm{NMPC}}=20$ elements and the MHE estimation horizon was set tp $N_{\mathrm{MHE}}=10$ elements.

The NMPC weighting matrices $Q$ and $R$ were chosen diagonal, with each entry specified in Table II, accordingly with the state it corresponds to. Note that the units of the weights are defined consistently with the variables they correspond to, so as to yield a dimensionless cost.

The MHE matrix $Q_{E}$ was chosen so as to match the variance of the measurement noise, given in Table I. Based on the same variance, Gaussian noise has been added to all measurements in the proposed simulations.

\section{B. Simulation Results}

In this Section, the simulation results obtained for the model proposed in Section II and the control algorithm proposed in Section III are presented.

The investigated scenario considers tracking a power optimal periodic reference trajectory in a turbulent wind. The reference trajectory and the simulated trajectory are
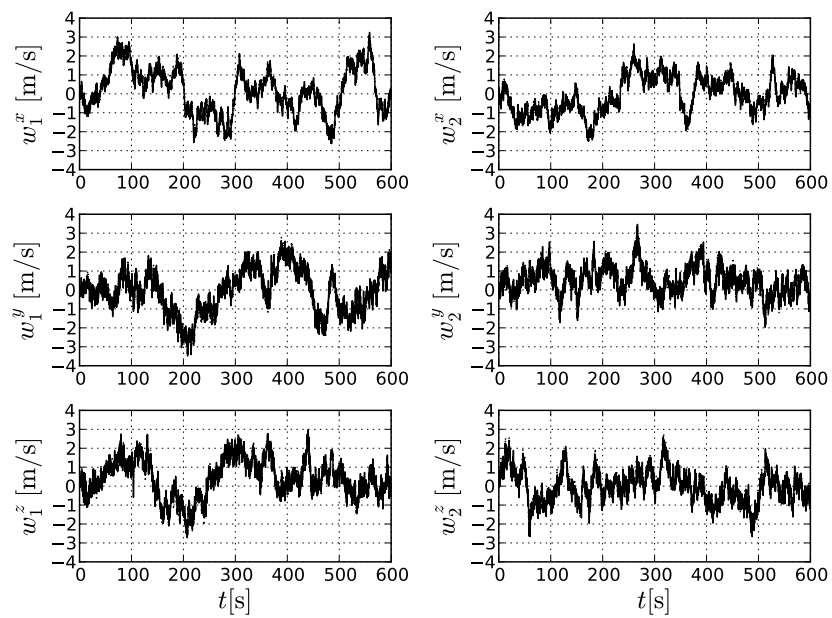

Fig. 2. Real wind turbulence in dashed line and turbulence wind speed estimated by the MHE in thick line for the two airfoils. As the estimation error is small, the dashed line is covered by the continuous line.

displayed in Figure 1. It can be seen that the airfoil is well stabilized around the reference even in the presence of strong turbulences.

The wind turbulences estimated by the MHE are displayed in Figure 2, where it can be seen that the estimates closely match the real turbulences. Note that the wind profile used in simulations has not been generated using the simple model described in Section II, but with a Von Karman model. Since is assumes a level flight at constant velocity, the Von Karman model is not the most appropriate turbulence model for AWE systems, and should not be used for certification purposes. Given the lack of wind models specific to AWE systems, the Von Karman wind model is still useful to test the control algorithm. In particular, it allows one to show that the component of a complex turbulent wind which is relevant to the system dynamics can still be well estimated with a simple model that does not rely on specific assumptions on the stochastic properties of the wind.

In particular, as shown in Figure 3, the power spectral density (PSD) of the estimation error is much lower than the one of the original signal at low frequencies. The signal-tonoise ratio (SNR) is thus high at low frequencies. At higher frequencies, where the SNR decreases, also the PSD of the turbulences is low.

For the purpose of illustrating the ability of NMPC to deal with constraints, the bounds on the control surfaces and their rates have been artificially tightened so as to activate them often in the simulation scenario. The resulting trajectories are displayed in Figure 4, together with the bounds.

The simulations were run on a $2.8 \mathrm{GHz} \mathrm{CPU}$ and the computational times are consistently below $T_{\mathrm{S}}=125 \mathrm{~ms}$, allowing for a real-time implementation.

\section{Conclusion \& Further Developments}

This paper has presented a control scheme for a dual airfoil AWE system in drag mode. The system model is a highly 

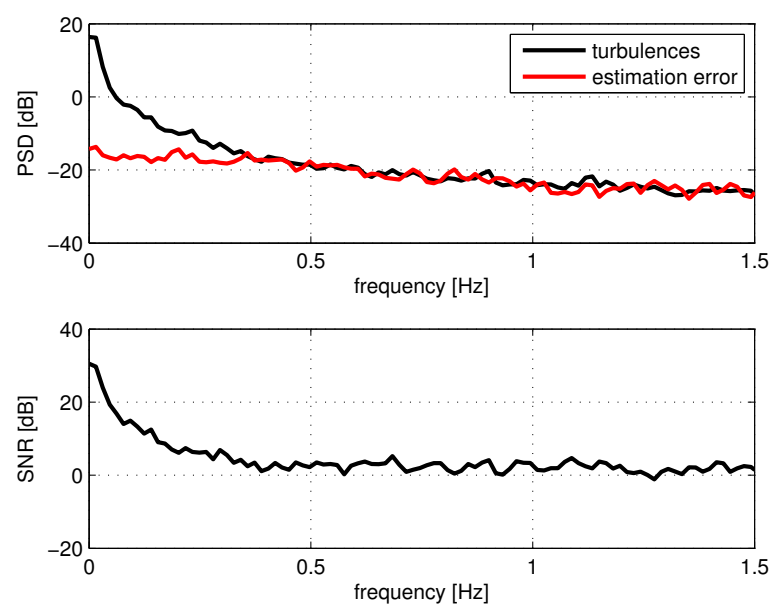

Fig. 3. Power spectral density of the turbulences and of the estimation error (top graph). Signal-to-noise ratio for the estimated signal (bottom graph).

nonlinear index 1 DAE which consists of 56 differential states, 3 algebraic states and 14 controls.

The computation of the reference trajectory involves the solution of an involved periodic optimal control problem. In order to be able to solve this problem, particular care has been taken in the computation of a suitable initial guess.

The control technique, based on Nonlinear Model Predictive Control (NMPC) and Moving Horizon Estimation (MHE), was tested in turbulent wind simulations. The MHE scheme is able to estimate the state based on the fusion of the data coming from all sensors while also estimating the wind turbulences. Based on these estimates, the NMPC scheme stabilizes the system on the periodic reference trajectory while satisfying the imposed constraints. The efficient implementation of the optimization routines results in a scheme which is fast enough for a real-time implementation.

The choice of a tracking cost function guarantees that the system will be stabilized, but is suboptimal in the sense of maximizing the energy extracted from the airmass. Future research will aim at further investigating the stability properties of NMPC schemes based on cost functions that directly maximize the extracted energy.

Dual airfoil AWE systems can also operate in pumping mode. Future work will aim at developing a control scheme also for these systems.

\section{REFERENCES}

[1] J. Laks, L. Pao, and A. Wright, "Control of Wind Turbines: Past, Present, and Future," in American Control Conference, pp. 2096-2103, 2009.

[2] E. A. Bossanyi, "Further Load Reductions with Individual Pitch Control," Wind Energy, vol. 8, pp. 481-485, 2005.

[3] M. Loyd, "Crosswind Kite Power," Journal of Energy, vol. 4, pp. 106111, July 1980.

[4] M. Zanon, S. Gros, J. Andersson, and M. Diehl, "Airborne Wind Energy Based on Dual Airfoils," IEEE Transactions on Control Systems Technology, vol. 21, July 2013.

[5] M. Diehl, L. Magni, and G. D. Nicolao, "Efficient nmpc of unstable periodic systems using approximate infinite horizon closed loop costing," Annual Reviews in Control, vol. 28, no. 1, pp. 37 - 45, 2004.
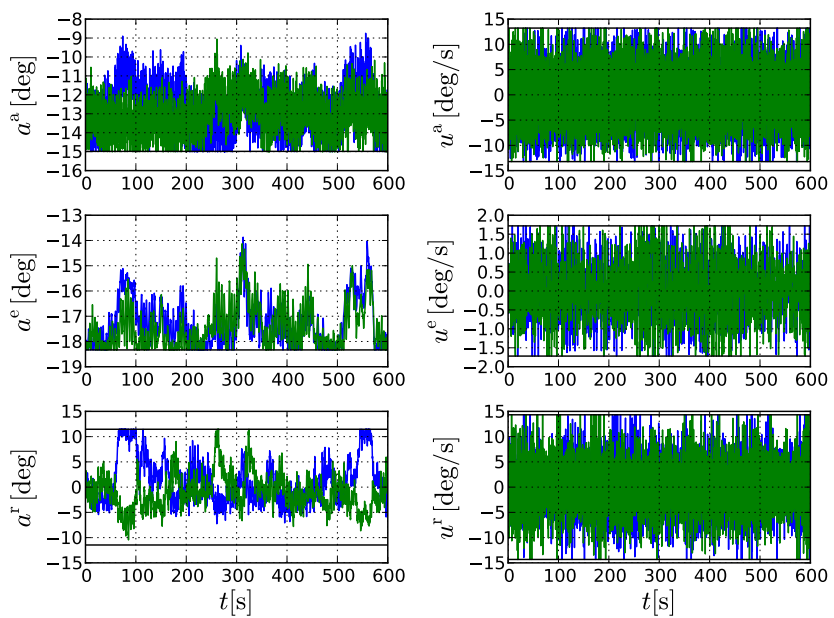

Fig. 4. Control surfaces and their time rates.

[6] B. Houska and M. Diehl, "Optimal Control for Power Generating Kites," in Proc. 9th European Control Conference, (Kos, Greece,), pp. 3560-3567, 2007. (CD-ROM).

[7] S. Gros, M. Zanon, and M. Diehl, "Orbit Control for a Power Generating Airfoil Based on Nonlinear MPC," in American Control Conference, 2012. (submitted).

[8] S. Gros, M. Zanon, and M. Diehl, "Control of Airborne Wind Energy Systems Based on Nonlinear Model Predictive Control \& Moving Horizon Estimation," in European Control Conference, 2013. (submitted)

[9] M. Zanon, S. Gros, and M. Diehl, "Rotational Start-up of Tethered Airplanes Based on Nonlinear MPC and MHE," in Proceedings of the European Control Conference, 2013. (accepted for publication).

[10] S. Gros, M. Zanon, and M. Diehl, "A Relaxation Strategy for the Optimization of Airborne Wind Energy Systems," in European Control Conference, 2013. (submitted).

[11] M. Diehl, H. Bock, J. Schlöder, R. Findeisen, Z. Nagy, and F. Allgöwer, "Real-time optimization and Nonlinear Model Predictive Control of Processes governed by differential-algebraic equations," Journal of Process Control, vol. 12, no. 4, pp. 577-585, 2002.

[12] B. Houska, H. Ferreau, and M. Diehl, "An Auto-Generated Real-Time Iteration Algorithm for Nonlinear MPC in the Microsecond Range," Automatica, vol. 47, no. 10, pp. 2279-2285, 2011.

[13] S. Gros, M. Zanon, M. Vukov, and M. Diehl, "Nonlinear MPC and MHE for Mechanical Multi-Body Systems with Application to Fast Tethered Airplanes," in Proceedings of the 4th IFAC Nonlinear Model Predictive Control Conference, Noordwijkerhout, The Netherlands, 2012.

[14] S. Gros and M. Diehl, Airborne Wind Energy, ch. Modeling of Airborne Wind Energy Systems in Natural Coordinates. Springer, 2013.

[15] Pamadi, Performance, Stability, Dynamics, and Control of Airplanes. American Institute of Aeronautics and Astronautics, Inc., 2003.

[16] M. Zanon, S. Gros, and M. Diehl, Airborne Wind Energy, ch. Control of Rigid-Airfoil Airborne Wind Energy Systems. Springer, 2013.

[17] G. Horn, S. Gros, and M. Diehl, Airborne Wind Energy. Springer, 2013

[18] L. Biegler, "An overview of simultaneous strategies for dynamic optimization," Chemical Engineering and Processing, vol. 46, pp. 10431053, 2007.

[19] H. Bock and K. Plitt, "A multiple shooting algorithm for direct solution of optimal control problems," in Proceedings 9th IFAC World Congress Budapest, pp. 242-247, Pergamon Press, 1984.

[20] D. Leineweber, I. Bauer, A. Schäfer, H. Bock, and J. Schlöder, "An Efficient Multiple Shooting Based Reduced SQP Strategy for LargeScale Dynamic Process Optimization (Parts I and II)," Computers and Chemical Engineering, vol. 27, pp. 157-174, 2003.

[21] M. Diehl, H. Bock, and J. Schlöder, "A real-time iteration scheme for nonlinear optimization in optimal feedback control," SIAM Journal on Control and Optimization, vol. 43, no. 5, pp. 1714-1736, 2005. 
[22] H. Ferreau, T. Kraus, M. Vukov, W. Saeys, and M. Diehl, "High-speed moving horizon estimation based on automatic code generation," in Proceedings of the 51th IEEE Conference on Decision and Control (CDC 2012), 2012.

[23] H. Ferreau, Model Predictive Control Algorithms for Applications with Millisecond Timescales. PhD thesis, K.U. Leuven, 2011. 